\title{
Exact Traveling Wave Solutions of Nonlinear PDEs in Mathematical Physics
}

\author{
Jameel F. Alzaidy \\ Mathematics Department, Faculty of Science, Taif University, Taif, KSA \\ Email: j-f-h-z@hotmail.com
}

Received April 27, 2012; revised May 27, 2012; accepted June 3, 2012

\begin{abstract}
In the present article, we construct the exact traveling wave solutions of nonlinear PDEs in mathematical physics via the variant Boussinesq equations and the coupled KdV equations by using the extended mapping method and auxiliary equation method. This method is more powerful and will be used in further works to establish more entirely new solutions for other kinds of nonlinear partial differential equations arising in mathematical physics.
\end{abstract}

Keywords: Extended Mapping Method; Auxiliary Equation Method; The Variant Boussinesq Equations; The Coupled KdV Equations; Traveling Wave Solutions

\section{Introduction}

The effort in finding exact solutions to nonlinear equations is important for the understanding of most nonlinear physical phenomena. For instance, the nonlinear wave phenomena observed in fluid dynamics, plasma and optical fibers are often modeled by the bell shaped sech solutions and the kink shaped tanh solutions. Many effective methods have been presented, such as inverse scattering transform method [1], Bäcklund transformation [2], Darboux transformation [3], Hirota bilinear method [4], variable separation approach [5], various tanh methods [6-9], homogeneous balance method [10], similarity reductions method [11,12], $\left(G^{\prime} / G\right)$-expansion method [13], the reduction $\mathrm{mKdV}$ equation method [14], the tri-function method $[15,16]$, the projective Riccati equation method [17], the Weierstrass elliptic function method [18], the Sine-Cosine method $[19,20]$, the Jacobi elliptic function expansion [21,22], the complex hyperbolic function method [23], the truncated Painleve' expansion [24], the F-expansion method [25], the rank analysis method [26], the ansatz method [27,28], the exp-function expansion method [29], the sub-ODE method [30], and so on.

The main objective of this paper is used the extended mapping method and auxiliary equation method to construct the exact solutions for nonlinear evolution equations in the mathematical physics via the variant Boussinesq equations and the coupled $\mathrm{KdV}$ equations.

\section{Description of the Extended Mapping Method}

Suppose we have the following nonlinear PDE:

$$
F\left(u, u_{t}, u_{x}, u_{t t}, u_{x x}, u_{x t}, \cdots\right)=0,
$$

where $u=u(x, t)$ is an unknown function, $F$ is a polynomial in $u=u(x, t)$ and its various partial derivatives in which the highest order derivatives and nonlinear terms are involved. In the following we give the main steps of a deformation method:

Step 1. The traveling wave variable

$$
u(x, t)=u(\xi), \xi=k(x-\omega t),
$$

where $k$ and $\omega$ are the wave number and the wave speed, respectively. Under the transformation (2), Equation (1) becomes an ordinary differential equation (ODE) as

$$
P\left(u,-k \omega u^{\prime}, k u^{\prime}, k^{2} \omega^{2} u^{\prime \prime}, k^{2} u^{\prime \prime}, \cdots\right)=0,
$$

where ' $=\frac{d}{d \xi}$.

Step 2. Suppose that the solution Equation (3) has the following form:

$$
\begin{aligned}
u(\xi)= & a_{0}+\sum_{i=1}^{n}\left(a_{i} f^{i}(\xi)+b_{i} f^{-i}(\xi)\right) \\
& +\sum_{i=2}^{n} c_{i} f^{i-2}(\xi) f^{\prime}(\xi) \\
& +\sum_{i=-1}^{n} d_{i} f^{i}(\xi) f^{\prime}(\xi),
\end{aligned}
$$

where $a_{0}, a_{i}, b_{i}, c_{i}$ and $d_{i}$ are constants to be determined later, while $f(\xi)$ satisfies the auxiliary ordinary differential equation:

$$
\left[f^{\prime}(\xi)\right]^{2}=p f^{4}(\xi)+q f^{2}(\xi)+r,
$$


where $p, q$ and $r$ are arbitrary constants.

Step 3. The positive integer " $n$ " can be determined by considering the homogeneous balance between the highest derivative term and the nonlinear terms appearing in Equation (3). Therefore, we can get the value of $n$ in Equation (4).

Step 4. Substituting Equation (4) into Equation (3) with the condition (5), we obtain polynomial in $f^{i}(\xi)\left[f^{\prime}(\xi)\right]^{j}, \quad(i=\cdots,-2,-1,0,1,2, \cdots ; j=0,1)$. Setting each coefficient of this polynomial to be zero, yields a set of algebraic equations for $a_{0}, a_{i}, b_{i}, c_{i}, d_{i}, \omega$ and $k$.

Step 5. Solving the algebraic equations by use of Maple or Mathematica, we have $a_{0}, a_{i}, b_{i}, c_{i}, d_{i}$ and $k$ expressed by $p, q, r$.

Step 6. Since the general solutions of auxiliary Equation (5) have been well known for us (see Appendix A), then substituting the obtained coefficients and the general solution of Equation (5) into Equation (4), we have the travelling wave solutions of the nonlinear PDE (1).

\section{Applications of the Method}

In this section, we apply the extended mapping method to construct the exact solutions for the variant Boussinesq equations and the coupled $\mathrm{KdV}$ equations. Which are very important nonlinear evolution equations in mathematical physics and have been paid attention by many researchers.

\subsection{Example 1. The Variant Boussinesq Equations}

We start with the variant Boussinesq equations [31] in the following form:

$$
\begin{aligned}
& v_{t}=\alpha u_{x x x}-u_{x}-(u v)_{x}, \\
& u_{t}=3 \alpha u_{x x t}-v_{x}-u u_{x},
\end{aligned}
$$

where $\alpha$ is a constant. As models for water waves, $u$ is the velocity and $v$ is the total depth. Wang [32] obtained their solitary wave solutions by using homogeneous balance method while Fan et al. [33] got a series of new traveling wave solutions of this system by using an algebraic method. The traveling wave variables below

$$
u(x, t)=u(\xi), v(x, t)=v(\xi), \xi=k(x-\omega t),
$$

permit us converting the Equations (6) and (7) into ODEs for $u(x, t)=u(\xi)$ and $v(x, t)=v(\xi)$ as follows:

$$
-\omega v^{\prime}+u^{\prime}+(u v)^{\prime}-\alpha k^{2} u^{\prime \prime \prime}=0,
$$

and

$$
-\omega u^{\prime}+u u^{\prime}+v^{\prime}+3 \alpha k^{2} \omega u^{\prime \prime}=0,
$$

On integrating Equations (9) and (10) with respect to $\xi$ once, we get

$$
L_{1}-\omega v+u+u v-\alpha k^{2} u^{\prime \prime}=0,
$$

and

$$
L_{2}-\omega u+\frac{u^{2}}{2}+v+3 \alpha k^{2} \omega u^{\prime \prime}=0,
$$

where $L_{1}$ and $L_{2}$ are integration constants.

Suppose that the solutions of Equations (11) and (12) can be expressed by

$$
\begin{aligned}
u(\xi)= & a_{0}+\sum_{i=1}^{n}\left(a_{i} f^{i}(\xi)+b_{i} f^{-i}(\xi)\right) \\
& +\sum_{i=2}^{n} c_{i} f^{i-2}(\xi) f^{\prime}(\xi) \\
& +\sum_{i=-1}^{-n} d_{i} f^{i}(\xi) f^{\prime}(\xi), \\
v(\xi)= & \alpha_{0}+\sum_{i=1}^{m}\left(\alpha_{i} f^{i}(\xi)+\beta_{i} f^{-i}(\xi)\right) \\
& +\sum_{i=2}^{m} \gamma_{i} f^{i-2}(\xi) f^{\prime}(\xi) \\
& +\sum_{i=-1}^{-m} e_{i} f^{i}(\xi) f^{\prime}(\xi),
\end{aligned}
$$

where $a_{0}, a_{i}, b_{i}, c_{i}, d_{i}, \alpha_{0}, \alpha_{i}, \beta_{i}, \gamma_{i}$, and $e_{i}$ are constants to be determined later.

Considering the homogeneous balance between the highest order derivatives and the nonlinear terms in Equations (11) and (12), we get $n=m=2$, hence the exact solutions of Equations (11) and (12) can be rewritten as:

$$
\begin{aligned}
u(\xi)= & a_{0}+a_{1} f(\xi)+b_{1} \frac{1}{f(\xi)}+a_{2} f^{2}(\xi)+b_{2} \frac{1}{f^{2}(\xi)} \\
& +c_{2} f^{\prime}(\xi)+d_{1} \frac{f^{\prime}(\xi)}{f(\xi)}+d_{2} \frac{f^{\prime}(\xi)}{f^{2}(\xi)}
\end{aligned}
$$

and

$$
\begin{aligned}
v(\xi)= & \alpha_{0}+\alpha_{1} f(\xi)+\beta_{1} \frac{1}{f(\xi)}+\alpha_{2} f^{2}(\xi)+\beta_{2} \frac{1}{f^{2}(\xi)} \\
& +\gamma_{2} f^{\prime}(\xi)+e_{1} \frac{f^{\prime}(\xi)}{f(\xi)}+e_{2} \frac{f^{\prime}(\xi)}{f^{2}(\xi)},
\end{aligned}
$$

where $a_{0}, a_{1}, a_{2}, b_{1}, b_{2}, c_{2}, d_{1}, d_{2}, \alpha_{0}, \alpha_{1}, \alpha_{2}, \beta_{1}, \beta_{2}, \gamma_{2}, e_{1}$ and $e_{2}$ are constants to be determined later. Substituting Equations (15) and (16) with the condition (5) into Equations (11) and (12) and collecting all terms with the same power of $f^{i}(\xi)\left[f^{\prime}(\xi)\right]^{j}$,

$(i=\cdots,-2,-1,0,1,2, \cdots ; j=0,1)$. Setting each coefficients of this polynomial to be zero, we get a system of algebraic equations which can be solved by Maple or Mathe- 
matica. Thus we obtain the two sets of solutions as:

\section{Case 1:}

$a_{0}=a_{1}=b_{1}=b_{2}=d_{1}=d_{2}=\alpha_{1}=\beta_{1}=\beta_{2}=e_{1}=e_{2}=0$,

$a_{2}=-\frac{p\left(1+6 p \omega^{2}\right)}{q \omega}, c_{2}=-\frac{\sqrt{p}\left(1+6 \omega^{2}\right)}{q \omega}$,

$\alpha_{0}=\frac{1}{18}\left(-15+\frac{1}{\omega^{2}}\right), \alpha_{2}=\frac{p\left(1+6 \omega^{2}\right)}{6 q \omega^{2}}$,

$\gamma_{2}=\frac{\sqrt{p}\left(1+6 \omega^{2}\right)}{6 q \omega^{2}}, L_{1}=\frac{q^{2} \omega^{2}\left(1-15 \omega^{2}\right)+p r\left(1+6 \omega^{2}\right)^{2}}{18 q^{2} \omega^{3}}$,

$L_{2}=\frac{-3 p r\left(1+6 \omega^{2}\right)^{2}+q^{2}\left(15 \omega^{2}-1\right)}{18 q^{2} \omega^{2}}, k= \pm \frac{1}{3 \omega} \sqrt{\frac{1+6 \omega^{2}}{2 q \alpha}}$.

\section{Case 2:}

$a_{0}=a_{1}=b_{1}=a_{2}=c_{2}=d_{1}=\alpha_{1}=\beta_{1}=\alpha_{2}=e_{1}=0$,

$b_{2}=-\frac{r\left(1+6 r \omega^{2}\right)}{q \omega}, d_{2}=\frac{\sqrt{r}\left(1+6 \omega^{2}\right)}{q \omega}$,

$\alpha_{0}=\frac{1}{18}\left(-15+\frac{1}{\omega^{2}}\right), \beta_{2}=\frac{r\left(1+6 \omega^{2}\right)}{6 q \omega^{2}}$,

$e_{2}=-\frac{\sqrt{r}\left(1+6 \omega^{2}\right)}{6 q \omega^{2}}, L_{1}=\frac{q^{2} \omega^{2}\left(1-15 \omega^{2}\right)+p r\left(1+6 \omega^{2}\right)^{2}}{18 q^{2} \omega^{3}}$,

$L_{2}=\frac{-3 p r\left(1+6 \omega^{2}\right)^{2}+q^{2}\left(15 \omega^{2}-1\right)}{18 q^{2} \omega^{2}}, k= \pm \frac{1}{3 \omega} \sqrt{\frac{1+6 \omega^{2}}{2 q \alpha}}$.

Note that, there are other cases which are omitted here. Since the solutions obtained here are so many, we just list some of the exact solutions corresponding to Case 2 to illustrate the effectiveness of the extended mapping method.

Substituting (18) into Equation (15) and Equation (16) yields

$$
\begin{aligned}
u(\xi)= & -\frac{r\left(1+6 r \omega^{2}\right)}{q \omega}\left(\frac{1}{f^{2}(\xi)}\right) \\
& +\frac{\sqrt{r}\left(1+6 \omega^{2}\right)}{q \omega}\left(\frac{f^{\prime}(\xi)}{f^{2}(\xi)}\right),
\end{aligned}
$$

and

$$
\begin{aligned}
v(\xi)= & \frac{1}{18}\left(-15+\frac{1}{\omega^{2}}\right)+\frac{r\left(1+6 \omega^{2}\right)}{6 q \omega^{2}}\left(\frac{1}{f^{2}(\xi)}\right) \\
& -\frac{\sqrt{r}\left(1+6 \omega^{2}\right)}{6 q \omega^{2}}\left(\frac{f^{\prime}(\xi)}{f^{2}(\xi)}\right),
\end{aligned}
$$

where

$$
\xi= \pm \frac{1}{3 \omega} \sqrt{\frac{1+6 \omega^{2}}{2 q \alpha}}(x-\omega t) .
$$

According to the Appendix A, we have the following families of exact solutions:

Family 1. If $r=1, q=-\left(1+m^{2}\right), p=m^{2}, \quad f(\xi)=$ $\operatorname{sn}(\xi)$, then we get

$$
u(\xi)=\frac{\left(1+6 \omega^{2}\right)}{\left(1+m^{2}\right) \omega}\left[n s^{2}(\xi)-c s(\xi) d s(\xi)\right]
$$

and

$$
\begin{aligned}
v(\xi)= & \frac{1}{18}\left(-15+\frac{1}{\omega^{2}}\right) \\
& -\frac{\left(1+6 \omega^{2}\right)}{6 \omega^{2}\left(1+m^{2}\right)}\left[n s^{2}(\xi)-c s(\xi) d s(\xi)\right],
\end{aligned}
$$

where

$$
\xi= \pm \frac{i}{3 \omega} \sqrt{\frac{1+6 \omega^{2}}{2 \alpha\left(1+m^{2}\right)}}(x-\omega t) .
$$

Family 2. If $r=1-m^{2}, q=2 m^{2}-1, p=-m^{2}$, $f(\xi)=c n(\xi)$, then we get

$$
\begin{aligned}
u(\xi) & =\frac{\left(m^{2}-1\right)\left[1+6 \omega^{2}\left(1-m^{2}\right)\right]}{\left(2 m^{2}-1\right) \omega} n c^{2}(\xi) \\
& -\frac{\sqrt{1-m^{2}}\left(1+6 \omega^{2}\right)}{\left(2 m^{2}-1\right) \omega} d c(\xi) s c(\xi),
\end{aligned}
$$

and

$$
\begin{aligned}
v(\xi) & =\frac{1}{18}\left(-15+\frac{1}{\omega^{2}}\right)+\frac{\left(1-m^{2}\right)\left(1+6 \omega^{2}\right)}{6 \omega^{2}\left(2 m^{2}-1\right)} n c^{2}(\xi) \\
& +\frac{\sqrt{1-m^{2}}\left(1+6 \omega^{2}\right)}{6 \omega^{2}\left(2 m^{2}-1\right)} d c(\xi) s c(\xi),
\end{aligned}
$$

where

$$
\xi= \pm \frac{1}{3 \omega} \sqrt{\frac{1+6 \omega^{2}}{2 \alpha\left(2 m^{2}-1\right)}}(x-\omega t) .
$$

Family 3. If $r=m^{2}-1, q=2-m^{2}, p=-1, \quad f(\xi)=$ $d n(\xi)$, then we get

$$
\begin{aligned}
u(\xi)= & \frac{\left(1-m^{2}\right)\left[1+6 \omega^{2}\left(m^{2}-1\right)\right]}{\left(2-m^{2}\right) \omega} n d^{2}(\xi) \\
& -\frac{m^{2} \sqrt{m^{2}-1}\left(1+6 \omega^{2}\right)}{\left(2-m^{2}\right) \omega} \operatorname{sd}(\xi) c d(\xi),
\end{aligned}
$$

and 


$$
\begin{aligned}
v(\xi)= & \frac{1}{18}\left(-15+\frac{1}{\omega^{2}}\right)+\frac{\left(m^{2}-1\right)\left(1+6 \omega^{2}\right)}{6 \omega^{2}\left(2-m^{2}\right)} n d^{2}(\xi) \\
& +\frac{m^{2} \sqrt{m^{2}-1}\left(1+6 \omega^{2}\right)}{6 \omega^{2}\left(2-m^{2}\right)} \operatorname{sd}(\xi) c d(\xi),
\end{aligned}
$$

where

$$
\xi= \pm \frac{1}{3 \omega} \sqrt{\frac{1+6 \omega^{2}}{2 \alpha\left(2-m^{2}\right)}}(x-\omega t) .
$$

Family 4. If $r=m^{2}, \quad q=-\left(1+m^{2}\right), \quad p=1, \quad f(\xi)=$ $d c(\xi)$, then we get

$$
\begin{aligned}
u(\xi)= & \frac{m^{2}\left(1+6 r \omega^{2}\right)}{\omega\left(1+m^{2}\right)} c d^{2}(\xi) \\
& -\frac{m\left(1-m^{2}\right)\left(1+6 \omega^{2}\right)}{\omega\left(1+m^{2}\right)} \operatorname{sd}(\xi) n d(\xi),
\end{aligned}
$$

and

$$
\begin{aligned}
v(\xi)= & \frac{1}{18}\left(-15+\frac{1}{\omega^{2}}\right)-\frac{m^{2}\left(1+6 \omega^{2}\right)}{6 \omega^{2}\left(1+m^{2}\right)} c d^{2}(\xi) \\
& +\frac{m\left(1-m^{2}\right)\left(1+6 \omega^{2}\right)}{6 \omega^{2}\left(1+m^{2}\right)} \operatorname{sd}(\xi) n d(\xi),
\end{aligned}
$$

where

$$
\xi= \pm \frac{i}{3 \omega} \sqrt{\frac{1+6 \omega^{2}}{2 \alpha\left(1+m^{2}\right)}}(x-\omega t) .
$$

Family 5. If $r=1, q=2-m^{2}, p=1-m^{2}, f(\xi)=$ $s c(\xi)$, then we get

$$
u(\xi)=-\frac{\left(1+6 \omega^{2}\right)}{\omega\left(2-m^{2}\right)}\left[c s^{2}(\xi)-d s(\xi) n s(\xi)\right],
$$

and

$$
\begin{aligned}
v(\xi) & =\frac{1}{18}\left(-15+\frac{1}{\omega^{2}}\right) \\
& +\frac{\left(1+6 \omega^{2}\right)}{6 \omega^{2}\left(2-m^{2}\right)}\left[c s^{2}(\xi)-d s(\xi) n s(\xi)\right],
\end{aligned}
$$

where

$$
\xi= \pm \frac{1}{3 \omega} \sqrt{\frac{1+6 \omega^{2}}{2 \alpha\left(2-m^{2}\right)}}(x-\omega t) .
$$

Family 6. If $r=\frac{1}{4}, q=\frac{1}{2}\left(1-2 m^{2}\right), p=\frac{1}{4}, \quad f(\xi)=$ $n s(\xi) \pm c s(\xi)$, then we get

$$
\begin{aligned}
u(\xi)= & -\frac{\left(2+3 \omega^{2}\right)}{4 \omega\left(1-2 m^{2}\right)[n s(\xi) \pm c s(\xi)]^{2}} \\
& -\frac{\left(1+6 \omega^{2}\right) d n(\xi)}{\omega\left(1-2 m^{2}\right)[c n(\xi) \pm 1]}
\end{aligned}
$$

and

$$
\begin{aligned}
v(\xi)= & \frac{1}{18}\left(-15+\frac{1}{\omega^{2}}\right) \\
& +\frac{\left(1+6 \omega^{2}\right)}{12 \omega^{2}\left(1-2 m^{2}\right)[n s(\xi) \pm c s(\xi)]^{2}} \\
& +\frac{\left(1+6 \omega^{2}\right) d n(\xi)}{6 \omega^{2}\left(1-2 m^{2}\right)[c n(\xi) \pm 1]}
\end{aligned}
$$

where

$$
\xi= \pm \frac{1}{3 \omega} \sqrt{\frac{1+6 \omega^{2}}{\alpha\left(1-2 m^{2}\right)}}(x-\omega t) .
$$

Similarly, we can write down the other families of exact solutions of Equations (6) and (7) which are omitted for convenience.

\subsection{Example 2. The Coupled KdV Equations}

In this subsection, we consider the coupled KdV Equations [34]:

$$
\begin{gathered}
u_{t}=6 v v_{x}-6 \alpha u u_{x}-\alpha u_{x x x}, \\
v_{t}=-3 \alpha u v_{x}-\alpha v_{x x x},
\end{gathered}
$$

where $\alpha$ is a constant. The traveling wave variables (8) permit us converting the Equations (40) and (41) into ODEs in the forms:

$$
\begin{gathered}
\omega u^{\prime}+6 v v^{\prime}-6 \alpha u u^{\prime}-\alpha k^{2} u^{\prime \prime \prime}=0, \\
\omega v^{\prime}-3 \alpha u v^{\prime}-\alpha k^{2} v^{\prime \prime \prime}=0 .
\end{gathered}
$$

Suppose that the solutions of Equations (42) and (43) can be expressed by

$$
\begin{aligned}
u(\xi)= & a_{0}+\sum_{i=1}^{n}\left(a_{i} f^{i}(\xi)+b_{i} f^{-i}(\xi)\right) \\
& +\sum_{i=2}^{n} c_{i} f^{i-2}(\xi) f^{\prime}(\xi) \\
& +\sum_{i=-1}^{-n} d_{i} f^{i}(\xi) f^{\prime}(\xi), \\
v(\xi)= & \alpha_{0}+\sum_{i=1}^{m}\left(\alpha_{i} f^{i}(\xi)+\beta_{i} f^{-i}(\xi)\right) \\
& +\sum_{i=2}^{m} \gamma_{i} f^{i-2}(\xi) f^{\prime}(\xi) \\
& +\sum_{i=-1}^{-m} e_{i} f^{i}(\xi) f^{\prime}(\xi),
\end{aligned}
$$


where $a_{0}, a_{i}, b_{i}, c_{i}, d_{i}, \alpha_{0}, \alpha_{i}, \beta_{i}, \gamma_{i}$ and $e_{i}$ are constants to be determined later.

Considering the homogeneous balance between the highest order derivatives and the nonlinear terms in Equation (63) and Equation (64), we get $n=m=2$, so Equations (44) and (45) can be rewritten as:

$$
\begin{aligned}
u(\xi)= & a_{0}+a_{1} f(\xi)+b_{1} \frac{1}{f(\xi)}+a_{2} f^{2}(\xi)+b_{2} \frac{1}{f^{2}(\xi)} \\
& +c_{2} f^{\prime}(\xi)+d_{1} \frac{f^{\prime}(\xi)}{f(\xi)}+d_{2} \frac{f^{\prime}(\xi)}{f^{2}(\xi)}, \\
v(\xi)= & \alpha_{0}+\alpha_{1} f(\xi)+\beta_{1} \frac{1}{f(\xi)}+\alpha_{2} f^{2}(\xi)+\beta_{2} \frac{1}{f^{2}(\xi)} \\
& +\gamma_{2} f^{\prime}(\xi)+e_{1} \frac{f^{\prime}(\xi)}{f(\xi)}+e_{2} \frac{f^{\prime}(\xi)}{f^{2}(\xi)},
\end{aligned}
$$

where $a_{0}, a_{1}, a_{2}, b_{1}, b_{2}, c_{2}, d_{1}, d_{2}, \alpha_{0}, \alpha_{1}, \alpha_{2}, \beta_{1}, \beta_{2}, \gamma_{2}, e_{1}$ and $e_{2}$ are constants to be determined later. Substituting Equation (46) and Equation (47) with the condition (5) into Equation (42) and Equation (43) and collecting all terms with the same power of $f^{i}(\xi)\left[f^{\prime}(\xi)\right]^{j}$, $(i=\cdots,-2,-1,0,1,2, \cdots ; j=0,1)$. Setting each coefficients of this polynomial to be zero, we get a system of algebraic equations which can be solved by Maple or Mathematica to get the following sets of solutions:

\section{Case 1:}

$$
\begin{aligned}
a_{0} & =a_{1}=b_{1}=c_{2}=d_{1}=d_{2}=\alpha_{0}=\alpha_{1} \\
& =\beta_{1}=\gamma_{2}=e_{1}=e_{2}=0, \\
a_{2} & =-\frac{\omega p}{q \alpha}, b_{2}=-\frac{\omega r}{q \alpha}, \alpha_{2}=\frac{\omega p}{q \sqrt{2 \alpha}}, \\
\beta_{2} & =\frac{\omega r}{q \sqrt{2 \alpha}}, k= \pm \frac{1}{2} \sqrt{\frac{\omega}{\alpha q}} .
\end{aligned}
$$

\section{Case 2:}

$$
\begin{aligned}
a_{0} & =a_{1}=b_{1}=b_{2}=d_{1}=d_{2}=\alpha_{0}=\alpha_{1} \\
& =\beta_{1}=\beta_{2}=e_{1}=e_{2}=0, \\
a_{2} & =-\frac{2 \omega p}{q \alpha}, c_{2}=\frac{2 \omega \sqrt{p}}{q \alpha}, \alpha_{2}=-\frac{\omega p}{q} \sqrt{\frac{2}{\alpha}}, \\
\gamma_{2} & =\frac{\omega}{q} \sqrt{\frac{2 p}{\alpha}}, k= \pm \sqrt{\frac{\omega}{q \alpha}} .
\end{aligned}
$$

Note that, there are other cases which are omitted here. Since the solutions obtained here are so many, we just list some of the exact solutions corresponding to Case 2 to illustrate the effectiveness of the extended mapping method.

Substituting (49) into Equation (46) and Equation (47) yields

$$
u(\xi)=-\frac{2 \omega p}{q \alpha} f^{2}(\xi)+\frac{2 \omega \sqrt{p}}{q \alpha} f^{\prime}(\xi),
$$

and

$$
v(\xi)=-\frac{\omega p}{q} \sqrt{\frac{2}{\alpha}} f^{2}(\xi)+\frac{\omega}{q} \sqrt{\frac{2 p}{\alpha}} f^{\prime}(\xi),
$$

where

$$
\xi= \pm \sqrt{\frac{\omega}{q \alpha}}(x-\omega t) .
$$

According to the Appendix A, we have the following families of exact solutions:

Family 1. If $r=1, q=2 m^{2}-1, p=m^{2}\left(m^{2}-1\right)$, $f(\xi)=s d(\xi)$, then we get

$$
\begin{aligned}
u(\xi)= & \frac{2 \omega m^{2}\left(1-m^{2}\right)}{\alpha\left(2 m^{2}-1\right)} s d^{2}(\xi) \\
& +\frac{2 \omega m \sqrt{m^{2}-1}}{\alpha\left(2 m^{2}-1\right)} n d(\xi) c d(\xi),
\end{aligned}
$$

and

$$
\begin{aligned}
v(\xi)= & \frac{\omega m^{2}\left(1-m^{2}\right)}{\left(2 m^{2}-1\right)} \sqrt{\frac{2}{\alpha}} s d^{2}(\xi) \\
& +\frac{\omega m}{\left(2 m^{2}-1\right)} \sqrt{\frac{2\left(m^{2}-1\right)}{\alpha}} n d(\xi) c d(\xi),
\end{aligned}
$$

where

$$
\xi= \pm \sqrt{\frac{\omega}{\alpha\left(2 m^{2}-1\right)}}(x-\omega t) .
$$

Family 2. If $r=m^{2}\left(m^{2}-1\right), q=2 m^{2}-1, p=1$, $f(\xi)=d s(\xi)$, then we get

$$
u(\xi)=-\frac{2 \omega}{\alpha\left(2 m^{2}-1\right)}\left[d s^{2}(\xi)+c s(\xi) n s(\xi)\right],
$$

and

$$
v(\xi)=-\frac{\omega}{2 m^{2}-1} \sqrt{\frac{2}{\alpha}}\left[d s^{2}(\xi)+c s(\xi) n s(\xi)\right],
$$

where

$$
\xi= \pm \sqrt{\frac{\omega}{\alpha\left(2 m^{2}-1\right)}}(x-\omega t)
$$

Family 3. If $r=\frac{m^{2}}{4}, q=\frac{1}{2}\left(m^{2}-2\right), p=\frac{m^{2}}{4}$, $f(\xi)=\operatorname{sn}(\xi) \pm i c n(\xi)$, then we get 


$$
\begin{aligned}
u(\xi)= & -\frac{\omega m^{2}}{\alpha\left(m^{2}-2\right)}[\operatorname{sn}(\xi) \pm i c n(\xi)]^{2} \\
& +\frac{2 \omega m}{\alpha\left(m^{2}-2\right)}[\operatorname{cn}(\xi) d n(\xi) \mp i s n(\xi) d n(\xi)],
\end{aligned}
$$

and

$$
\begin{aligned}
v(\xi)= & -\frac{\omega m^{2}}{m^{2}-2} \sqrt{\frac{2}{\alpha}}[\operatorname{sn}(\xi) \pm i c n(\xi)]^{2} \\
& +\frac{\omega m}{m^{2}-2} \sqrt{\frac{2}{\alpha}}[\operatorname{cn}(\xi) d n(\xi) \mp i s n(\xi) d n(\xi)],
\end{aligned}
$$

where

$$
\xi= \pm \sqrt{\frac{2 \omega}{\alpha\left(m^{2}-2\right)}}(x-\omega t) .
$$

Family 4. If $r=1, q=-\left(1+m^{2}\right), p=m^{2}, f(\xi)=$ $\operatorname{sn}(\xi)$, then we get

$$
\begin{aligned}
u(\xi)= & \frac{2 \omega m^{2}}{\alpha\left(1+m^{2}\right)} s n^{2}(\xi) \\
& -\frac{2 \omega m}{\alpha\left(1+m^{2}\right)} \operatorname{cn}(\xi) d n(\xi),
\end{aligned}
$$

and

$$
\begin{aligned}
v(\xi)= & \frac{\omega m^{2}}{1+m^{2}} \sqrt{\frac{2}{\alpha}} s n^{2}(\xi) \\
& -\frac{\omega m}{1+m^{2}} \sqrt{\frac{2}{\alpha}} c n(\xi) d n(\xi),
\end{aligned}
$$

where

$$
\xi= \pm i \sqrt{\frac{\omega}{\alpha\left(1+m^{2}\right)}}(x-\omega t) .
$$

Family 5. If $r=1-m^{2}, q=2 m^{2}-1, p=-m^{2}$, $f(\xi)=c n(\xi)$, then we get

$$
\begin{aligned}
u(\xi)= & \frac{2 \omega m^{2}}{\alpha\left(2 m^{2}-1\right)} c n^{2}(\xi) \\
& -\frac{2 i m \omega}{\alpha\left(2 m^{2}-1\right)} \operatorname{sn}(\xi) d n(\xi),
\end{aligned}
$$

and

$$
\begin{aligned}
v(\xi)= & \frac{\omega m^{2}}{2 m^{2}-1} \sqrt{\frac{2}{\alpha}} c n^{2}(\xi) \\
& -\frac{i m \omega}{2 m^{2}-1} \sqrt{\frac{2}{\alpha}} \operatorname{sn}(\xi) d n(\xi),
\end{aligned}
$$

where

$$
\xi= \pm \sqrt{\frac{\omega}{\alpha\left(2 m^{2}-1\right)}}(x-\omega t) .
$$

Family 6. If $r=1-m^{2}, q=2-m^{2}, p=1, f(\xi)=$ $\operatorname{cs}(\xi)$, then we get

$$
u(\xi)=-\frac{2 \omega}{\alpha\left(2-m^{2}\right)}\left[c s^{2}(\xi)+n s(\xi) d s(\xi)\right],
$$

and

$$
v(\xi)=-\frac{\omega}{2-m^{2}} \sqrt{\frac{2}{\alpha}}\left[c s^{2}(\xi)+n s(\xi) d s(\xi)\right],
$$

where

$$
\xi= \pm \sqrt{\frac{\omega}{\alpha\left(2-m^{2}\right)}}(x-\omega t) .
$$

\section{Conclusion and Discussion}

In this article, we have found the exact solutions of the variant Boussinesq equations and the coupled $\mathrm{KdV}$ equations by using the extended mapping method and the auxiliary equation method. Also, we conclude according to the Appendix B that our results in terms of Jacobi elliptic functions generate into hyperbolic functions when $m \rightarrow 1$ and generate into trigonometric functions when $m$ $\rightarrow 0$. This method provides a powerful mathematical tool to obtain more general exact solutions of a great many nonlinear PDEs in mathematical physics.

\section{REFERENCES}

[1] M. J. Ablowitz and P. A. Clarkson, "Soliton, Nonlinear Evolution Equations and Inverse Scattering," Cambridge University Press, Cambridge, 1991. doi:10.1017/CBO9780511623998

[2] C. H. Gu, et al., "Soliton Theory and Its Application," Zhejiang Science and Technology Press, Zhejiang, 1990.

[3] V. B. Matveev and M. A. Salle, "Darboux Transformation and Soliton," Springer, Berlin, 1991.

[4] R. Hirota, "The Direct Method in Soliton Theory," Cambridge University Press, Cambridge, 2004. doi:10.1017/CBO9780511543043

[5] S. Y. Lou and J. Z. Lu, "Special Solutions from Variable Separation Approach: Davey-Stewartson Equation," Journal of Physics A: Mathematical and General, Vol. 29, No. 14, 1996, pp. 4209-4215. doi:10.1088/0305-4470/29/14/038

[6] E. J. Parkes and B. R. Duffy, "Travelling Solitary Wave Solutions to a Compound KdV-Burgers Equation," Physics Letters A, Vol. 229, No. 4, 1997, pp. 217-220. doi:10.1016/S0375-9601(97)00193-X

[7] E. Fan, "Extended Tanh-Function Method and Its Applications to Nonlinear Equations," Physics Letters A, Vol. 277, No. 4-5, 2000, pp. 212-218. doi:10.1016/S0375-9601(00)00725-8

[8] Z. Y. Yan, "New Explicit Travelling Wave Solutions for Two New Integrable Coupled Nonlinear Evolution Equa- 
tions," Physics Letters A, Vol. 292, No. 1-2, 2001, pp. 100-106. doi:10.1016/S0375-9601(01)00772-1

[9] Y. Chen and Y. Zheng, "Generalized Extended TanhFunction Method to Construct New Explicit Exact Solutions for the Approximate Equations for Long Water Waves," International Journal of Modern Physics C, Vol. 14, No. 5, 2003, pp. 601-611. doi:10.1142/S0129183103004760

[10] M. L. Wang, “Application of a Homogeneous Balance Method to Exact Solutions of Nonlinear Equations in Mathematical Physics," Physics Letters A, Vol. 216, No. 1-5, 1996, pp. 67-75. doi:10.1016/0375-9601(96)00283-6

[11] G. W. Bluman and S. Kumei, "Symmetries and Differential Equations," Springer-Verlag, New York, 1989.

[12] P. J. Olver, "Applications of Lie Groups to Differential Equations," Springer-Verlag, New York, 1986. doi:10.1007/978-1-4684-0274-2

[13] E. M. E. Zayed and K. A. Gepreel, "The $\left(\frac{G^{\prime}}{G}\right)$-Expansion Method for Finding Traveling Wave Solutions of Nonlinear PDEs in Mathematical Physics," Journal of Mathematical Physics, Vol. 50, No. 1, 2009, Article ID: 013502. doi:10.1063/1.3033750

[14] Z. Y. Yan, "A Reduction mKdV Method with Symbolic Computation to Constract New Doubly-Periodic Solutions for Nonlinear Wave Equations," International Journal of Modern Physics C, Vol. 14, No. 5, 2003, pp. 661-672. doi:10.1142/S0129183103004814

[15] Z. Y. Yan, "The New Tri-Function Method to Multiple Exact Solutions of Nonlinear Wave Equations," Physica Scripta, Vol. 78, No. 3, 2008, Article ID: 035001. doi:10.1088/0031-8949/78/03/035001

[16] Z. Y. Yan, "Periodic, Solitary and Rational Wave Solutions of the 3D Extended Quantum Zakharov-Kuznetsov Equation in Dense Quantum Plasmas," Physics Letters A, Vol. 373, No. 29, 2009, pp. 2432-2437. doi:10.1016/j.physleta.2009.04.018

[17] D. C. Lu and B. J. Hong, "New Exact Solutions for the (2+1)-Dimensional Generalized Broer-Kaup System," Applied Mathematics and Computation, Vol. 199, No. 2, 2008, pp. 572-580. doi:10.1016/j.amc.2007.10.012

[18] A. V. Porubov, "Periodical Solution to the Nonlinear Dissipative Equation for Surface Waves in a Convecting Liquid," Physics Letters A, Vol. 221, No. 6, 1996, pp. 391-394 doi:10.1016/0375-9601(96)00598-

[19] M. Wazwaz, "The Tanh and Sine-Cosine Method for Compact and Noncompact Solutions of Nonlinear Klein Gordon Equation," Applied Mathematics and Computation, Vol. 167, No. 2, 2005, pp. 1179-1195. doi:10.1016/j.amc.2004.08.006

[20] Z. Y. Yan and H. Q. Zhang, "New Explicit Solitary Wave Solutions and Periodic Wave Solutions for WhithamBroer-Kaup Equation in Shallow Water," Physics Letters A, Vol. 285, No. 5-6, 2001, pp. 355-362. doi:10.1016/S0375-9601(01)00376-0

[21] D. C. Lu, "Jacobi Elliptic Functions Solutions for Two Variant Boussinesq Equations," Chaos, Solitons and
Fractals, Vol. 24, No. 5, 2005, pp. 1373-1385. doi:10.1016/j.chaos.2004.09.085

[22] Z. Y. Yan, "Abundant Families of Jacobi Elliptic Functions of the $(2+1)$ Dimensional Integrable Davey-Stawartson-Type Equation via a New Method," Chaos, Solitons and Fractals, Vol. 18, No. 2, 2003, pp. 299-309. doi:10.1016/S0960-0779(02)00653-7

[23] C. L. Bai and H. Zhao, "Generalized Method to Construct the Solitonic Solutions to (3+1)-Dimensional Nonlinear Equation," Physics Letters A, Vol. 354, No. 5-6, 2006, pp. 428-436. doi:10.1016/j.physleta.2006.01.084

[24] F. Cariello and M. Tabor, "Similarity Reductions from Extended Painleve' Expansions for Nonintegrable Evolution Equations," Physica D: Nonlinear Phenomena, Vol. 53, No. 1, 1991, pp. 59-70. doi:10.1016/0167-2789(91)90164-5

[25] M. Wang and X. Li, "Extended F-Expansion and Periodic Wave Solutions for the Generalized Zakharov Equations," Physics Letters A, Vol. 343, No. 1-3, 2005, pp. 4854. doi:10.1016/i.physleta.2005.05.085

[26] X. Feng, "Exploratory Approach to Explicit Solution of Nonlinear Evolution Equations," International Journal of Theoretical Physics, Vol. 39, 2000, p. 222. doi:10.1023/A:1003615705115

[27] J. L. Hu, "Explicit Solutions to Three Nonlinear Physical Models," Physics Letters A, Vol. 287, No. 1-2, 2001, pp. 81-89. doi:10.1016/S0375-9601(01)00461-3

[28] J. L. Hu, “A New Method for Finding Exact Traveling Wave Solutions to Nonlinear Partial Differential Equations," Physics Letters A, Vol. 286, No. 2-3, 2001, pp. 175-179. doi:10.1016/S0375-9601(01)00291-2

[29] J. H. He and X. H. Wu, "Exp-Function Method for Nonlinear Wave Equations," Chaos, Solitons and Fractals, Vol. 30, No. 3, 2006, pp. 700-708. doi:10.1016/j.chaos.2006.03.020

[30] X. Z. Li and M. L. Wang, "A Sub-ODE Method for Finding Exact Solutions of a Generalized KdV-mKdV Equation with Higher Order Nonlinear Terms," Physics Letters A, Vol. 361, No. 1-2, 2007, pp. 115-118. doi:10.1016/j.physleta.2006.09.022

[31] E. M. E. Zayed and K. A. Gepreel, "Three Types of TravelingWave Solutions for Nonlinear Evolution Equations Using the $\left(\frac{G^{\prime}}{G}\right)$-Expansion Method," International Journal of Nonlinear Science, Vol. 7, 2009, p. 501.

[32] M. L. Wang, "Solitary Wave Solutions for Variant Boussinesq Equations," Physics Letters A, Vol. 199, No. 3-4, 1995, pp. 169-172. doi:10.1016/0375-9601(95)00092-H

[33] E. G. Fan and Y. C. Hon, "A Series of Travelling Wave Solutions for Two Variant Boussinesq Equations in Shallow Water Waves," Chaos, Solitons and Fractals, Vol. 15, No. 3, 2003, pp. 559-566. doi:10.1016/S0960-0779(02)00144-3

[34] R. Hirota and J. Satsuma, "Soliton Solutions of Coupled Korteweg-de Vries Equation," Physics Letters A, Vol. 85, No. 8-9, 1981, pp. 407-408. doi:10.1016/0375-9601(81)90423-0 
[35] K. A. Gepreel, "Exact Solutions for Nonlinear PDEs with the Variable Coefficients in Mathematical Physics," Jour- nal of Information and Computing Science, Vol. 6, No. 1, 2011, pp. 3-14.

\section{Appendix A}

The general solutions of the auxiliary Equation (5) and its derivatives [35] are listed as follows:

\begin{tabular}{ccccc}
\hline$p$ & $q$ & $r$ & $f(\xi)$ & $f^{\prime}(\xi)$ \\
\hline$m^{2}$ & $-\left(1+m^{2}\right)$ & 1 & $s n(\xi)$ & $c n(\xi) d n(\xi)$ \\
$-m^{2}$ & $2 m^{2}-1$ & $1-m^{2}$ & $c n(\xi)$ & $-s n(\xi) d n(\xi)$ \\
-1 & $2-m^{2}$ & $m^{2}-1$ & $d n(\xi)$ & $-m^{2} \operatorname{sn}(\xi) c n(\xi)$ \\
1 & $-\left(1+m^{2}\right)$ & $m^{2}$ & $d c(\xi)$ & $\left(1-m^{2}\right) n c(\xi) s c(\xi)$ \\
1 & $2-m^{2}$ & $1-m^{2}$ & $c s(\xi)$ & $-n s(\xi) d s(\xi)$ \\
$1-m^{2}$ & $2-m^{2}$ & 1 & $s c(\xi)$ & $n c(\xi) d c(\xi)$ \\
$m^{2}\left(m^{2}-1\right)$ & $2 m^{2}-1$ & 1 & $\operatorname{sd}(\xi)$ & $-c s(\xi) n s(\xi)$ \\
1 & $2 m^{2}-1$ & $m^{2}\left(m^{2}-1\right)$ & $d s(\xi)$ & $-d s(\xi) c s(\xi) \mp n s(\xi) d s(\xi)$ \\
$\frac{1}{4}$ & $\frac{1}{2}\left(1-2 m^{2}\right)$ & $\frac{1}{4}$ & $n s(\xi) \pm c s(\xi)$ & $c n(\xi) d n(\xi) \mp i \operatorname{sn}(\xi) d n(\xi)$ \\
$\frac{m^{2}}{4}$ & $\frac{1}{2}\left(m^{2}-2\right)$ & $\frac{m^{2}}{4}$ & $\operatorname{sn}(\xi) \pm i c n(\xi)$ & \\
\hline
\end{tabular}

where $0<m<1$ is the modulus of the Jacobi elliptic functions and $i=\sqrt{-1}$.

\section{Appendix B}

The Jacobi elliptic functions $s n(\xi), c n(\xi), d n(\xi), n s(\xi), c s(\xi), d s(\xi), s c(\xi), s d(\xi)$ generate into hyperbolic functions when $m \rightarrow 1$ as follows:
$\operatorname{sn}(\xi) \rightarrow \tanh (\xi)$
$\operatorname{cn}(\xi) \rightarrow \operatorname{sech}(\xi)$
$d n(\xi) \rightarrow \operatorname{sech}(\xi)$
$n s(\xi) \rightarrow \operatorname{coth}(\xi)$
$\operatorname{cs}(\xi) \rightarrow \operatorname{csch}(\xi)$
$d s(\xi) \rightarrow \operatorname{csch}(\xi)$
$s c(\xi) \rightarrow \sinh (\xi)$
$\operatorname{sd}(\xi) \rightarrow \sinh (\xi)$

and into trigonometric functions when $m \rightarrow 0$ as follows:

$\begin{array}{lccc}\operatorname{sn}(\xi) \rightarrow \sin (\xi) & c n(\xi) \rightarrow \cos (\xi) & n n(\xi) \rightarrow 1 & n s(\xi) \rightarrow \csc (\xi) \\ c s(\xi) \rightarrow \cot (\xi) & d s(\xi) \rightarrow \csc (\xi) & s c(\xi) \rightarrow \tan (\xi) & s d(\xi) \rightarrow \sin (\xi)\end{array}$

\section{Appendix C}
$n c(\xi)=\frac{1}{c n(\xi)}$
$n d(\xi)=\frac{1}{d n(\xi)}$
$c d(\xi)=\frac{c n(\xi)}{d n(\xi)}$
$d c(\xi)=\frac{d n(\xi)}{c n(\xi)}$
$\operatorname{cs}(\xi)=\frac{\operatorname{cn}(\xi)}{\operatorname{sn}(\xi)}$
$s c(\xi)=\frac{s n(\xi)}{c n(\xi)}$
$s d(\xi)=\frac{s n(\xi)}{d n(\xi)}$
$d s(\xi)=\frac{d n(\xi)}{s n(\xi)}$ 